\title{
Recent advances in the treatment of colorectal cancer: A review
}

Running title: Advances in colorectal cancer treatment

Seiichi Shinji, Takeshi Yamada, Akihisa Matsuda, Hiromichi Sonoda, Ryo Ohta,

Takuma Iwai, Koki Takeda, Kazuhide Yonaga, Yuka Masuda, Hiroshi Yoshida

Departments of Gastrointestinal and Hepato-Biliary-Pancreatic Surgery, Nippon Medical

School, Tokyo 113-8603, Japan

Address correspondence to:

Seiichi Shinji, M.D., Ph.D.

Departments of Gastrointestinal and Hepato-Biliary-Pancreatic Surgery, Nippon Medical

School, Tokyo 113-8603, Japan

Phone: +81-3-3822-2131 ext. (6752), Fax: +81-3-5685-0989

E-mail address: s-shinji@nms.ac.jp 


\begin{abstract}
Colorectal cancer (CRC) is the third most common cancer worldwide, and surgical treatment remains the first-line treatment to provide a cure. In addition to the aging population, obesity, low physical activity, and smoking habits increase CRC risk. Despite advances in surgical techniques, chemotherapy, and radiotherapy, colorectal cancer remains the second leading cause of cancer-related deaths worldwide. For early-stage $\mathrm{CRC}$, endoscopic treatment, including endoscopic mucosal resection and endoscopic submucosal dissection, has been performed. However, lymph node dissection is an integral part of surgical treatment for advanced-stage cancer because of the high incidence of lymph node metastasis. Conventional open surgery has evolved into laparoscopic and robotic surgery. Although prospective studies have confirmed the safety and feasibility of laparoscopic surgery for CRC, relevant treatment models of transverse colon cancer and rectal cancer still need to be further explored and validated. Furthermore, multidisciplinary treatment is needed to cure CRC completely. This review aimed to provide an update on recent advances in the surgical treatment of CRC.
\end{abstract}




\section{KEYWORDS}

colorectal cancer, surgery, endoscopy, chemotherapy, navigation 


\section{INTRODUCTION}

According to the International Agency for Research on Cancer (IARC) estimates in 2018, colorectal cancer (CRC) was the third most common cancer and the second leading cause of cancer-related mortality and is associated with high morbidity worldwide. ${ }^{1}$ Colorectal cancer (CRC) is a heterogeneous disease, and the majority of CRCs develop slowly from adenomatous polyps or adenomas. ${ }^{2}$ In addition to the adenoma to carcinoma sequence, approximately $25 \%$ of sporadic CRCs arise via serrated precursor lesions. ${ }^{3}$ The risk of developing CRC could also be associated with increasing age, male sex, genetic, environmental, socioeconomic status, nutritional status, physical activity, smoking, and lifestyle factors. ${ }^{4,5}$ Currently, the proportion of older adults with CRC has increased; in 2050, an estimated 6.9 million new CSCs will be diagnosed in adults aged 80 years or older worldwide (20.5\% of all cancer cases) ${ }^{6}$ In contrast, epidemiological studies have shown that the incidence of CRC in young people is also gradually increasing. ${ }^{7}$ In Japan, treatment for CRC is generally decided in accordance with the 2019 edition of the Japanese Society for Cancer of the Colon and Rectum (JSCCR) guidelines, which are prepared by the Japanese Society for Cancer of the Colon and Rectum. ${ }^{8}$ This review 
aimed to provide an update on recent advances in the treatment of CRC.

\section{ENDOSCOPIC TREATMENT}

Advances in the development of flexible endoscopes and endoscopic devices have increased the demand for minimally invasive treatments and expanded the indications for endoscopic treatments. ${ }^{9}$ Upon diagnosis, T1 CRC might be resected endoscopically in en bloc endoscopic mucosal resection (Fig.1) and endoscopic submucosal resection for large and complex lesions (Fig.2). Recently, peranal endoscopic myectomy has been performed for rectal lesions with severe fibrosis, in which dissection is performed between the inner circular and outer longitudinal muscle layers. ${ }^{10}$ Endoscopic full-thickness resection has also become possible (Fig.3); for lesions that are $<2 \mathrm{~cm}$ in size, this modality (performed using a full-thickness resection device system [Ovesco Endoscopy, Tuebingen, Germany]) has shown a good overall technical efficacy with acceptable safety. ${ }^{11}$ Endoscopic removal is both safer and less expensive than surgery when performed by expert endoscopists. ${ }^{12}$ In cases where endoscopic resection is challenging or the risk of complication is high due to various polyp-specific factors, such as size, location, 
appearance, and a sessile nature, where endoscopic resection is challenging or the risk of complication is high, a combined endoscopic and laparoscopic surgery (CELS) can significantly reduce costs and lower complication rates while preserving the patient's colon. ${ }^{13}$ Guidelines for treating T1 CRC in Japan mention lymphovascular invasion, histological grade, submucosal invasion depth, and tumor budding as risk factors for lymph node metastasis. ${ }^{8}$ Recently, Kudo et al. ${ }^{14}$ reported that an artificial intelligence system showed higher discriminating power than the current guideline for predicting lymph node metastasis in patients with T1 CRCs.

\section{SURGICAL TREATMENT}

Despite the rapid development of chemotherapy, radiotherapy, and immunotherapy, surgical resection remains the only possible cure for advanced-stage CRC. ${ }^{15}$ Therefore, it is particularly important to improve surgical treatment options for advanced CRC.

\section{The extent of lymph node dissection in advanced colon cancer}

Lymph node dissection, performed during surgical resection for CRC, is used for staging and has an essential impact on patient prognosis. The current standard of care for curative 
surgery for colon cancer is complete mesocolic excision (CME) with central vascular ligation (CVL) or D3 dissection. ${ }^{16}$ The concept of CME with CVL consists of sharp dissection along the embryological planes within the mesofascial interface with a full regional lymph node dissection, including central ligation of the supplying vessels. ${ }^{17}$ The embryologic fusion fascia of Toldt and Fredet is known landmark used by colorectal surgeons (Fig.4). Especially, in cases of right colon cancer, anatomical knowledge of the fusion fascia of Fredet is useful to achieve CME and D3 with reduced risk of intraoperative complications. ${ }^{18}$ Although meta-analysis shows that CME is a more effective strategy for improving specimen quality and survival, it involves a higher complication rate. ${ }^{19}$ The optimal surgery for colon cancer remains unclear because no evidence shows the beneficial impact of extensive (D3) versus more limited (D2) dissection oncological outcomes, which might increase morbidity. ${ }^{20}$ An international, prospective, observational cohort study (the T-REX study, $n=4,000$ patients) is being conducted to establish an international consensus on the extent of bowel resection and appropriate central lymphadenectomy in colon cancer surgery based on an in-depth analysis of lymph node mapping. ${ }^{21}$ 


\section{Laparoscopic surgery for advanced colon cancer}

The evolution of colorectal cancer surgery is shown in Figure 5. In the early 1990s, laparoscopic surgery was first proposed as an alternative to open surgery for lesions of the colon and rectum (Fig.5). ${ }^{22}$ Most randomized controlled trials in the United States and Europe reported short-term benefits compared with open surgery, with no significant differences in long-term outcome. ${ }^{23-28}$ Laparoscopic surgery for the treatment of colon cancer has been routinely performed worldwide. It is also recommended as an acceptable treatment option in Japan. ${ }^{29}$ However, patients with high BMI, clinical N2 and T4 disease, and tumor size $\geq 6 \mathrm{~cm}$ might require prudent selection of surgical approach because these groups tend to show worse survival than those who underwent open surgery. ${ }^{29}$ In contrast, laparoscopy is the dominant procedure, with significantly lower costs and lower surgical site infection than open surgery. ${ }^{30,} 31$ However, patient-reported outcomes (PROs), recognized endpoint after medical and surgical treatments, measures changes are similar between open and laparoscopic surgery in patients with colon cancer. ${ }^{32}$ Although prospective studies have confirmed the safety and feasibility of laparoscopic surgery for 
$\mathrm{CRC}$, the effectiveness of laparoscopic surgery for transverse colon cancer is not well established for anatomical reasons. The mesentery of the transverse colon is closely related to the proximal superior mesenteric artery and vein as well as to the foregut structures such as the greater omentum, the pancreas, and the lesser sac. ${ }^{33}$ In addition, there are many variations of vascular bifurcation, and the appropriate extent of lymph node dissection is not standardized; transverse colectomy is considered a complicated surgery. Against this background, there have been several reports on the usefulness of laparoscopic surgery for transverse colon cancer. Meta-analysis of transverse colectomy compared with open and laparoscopy provides similar survival benefits, earlier postoperative recovery, and shorter hospital stay. ${ }^{33}$ Furthermore, a randomized controlled trial showed similar short- and long-term outcomes of laparoscopic surgery for transverse colon cancer compared with open surgery. ${ }^{34}$ Laparoscopic extended right hemicolectomy and laparoscopic transverse colectomy offer similar oncological outcomes for midtransverse colon cancer. ${ }^{35}$ 


\section{Total mesorectal excision and lateral lymph node dissection for}

\section{lower rectal cancer}

Surgery for rectal cancer is more complex because of the accessibility and intricate anatomy of the pelvis. Total mesorectal excision (TME) is the standard oncological approach to rectal cancer because it contains most of the involved lymph nodes and tumor deposits, which was first proposed by Heald et al. ${ }^{36}$ in 1982 . This includes the routine excision of the intact mesorectum by precise, sharp dissection in the areolar tissue between the visceral and parietal layers of the pelvic fascia. ${ }^{37}$ A clear circumferential margin is generally defined as a distance greater than $1 \mathrm{~mm}$ between the tumor border and resection margin. ${ }^{38}$ Patients with involved circumferential margins have an increased risk of local recurrence and development of distant metastases. ${ }^{39,40}$ Contrastingly, lateral lymph node metastases exist in nearly $16 \%-23 \%$ of patients with rectal cancer, and $40 \%-$ $50 \%$ of patients with R0 resection reportedly achieved five-year survival. ${ }^{8}$ Management of lower rectal cancer has evolved differently in Japan compared to the rest of the world. For example, patients with stage II/III disease below the peritoneal reflection are commonly managed by TME or mesorectal excision (ME) and lateral lymph node 
dissection (LLND). ${ }^{41}$ However, western countries prefer to use TME plus preoperative chemoradiotherapy followed by TME and postoperative adjuvant chemotherapy with fluorouracil and oxaliplatin to reduce local recurrence. ${ }^{42}$ A large, randomized control trial, JCOG0212, showed no difference in relapse-free survival; however, local recurrence was significantly less common in the ME + LLND group, especially in the lateral pelvis. ${ }^{43}$ ME with LLND is recommended for patients with clinical stage III disease, whereas LLND can be omitted in patients with clinical stage II tumors. ${ }^{44}$ Furthermore, a metaanalysis comparing the TME plus LLND and TME only groups in rectal cancer patients showed no statistically significant differences in the overall survival and disease-free survival. ${ }^{45}$ Recently, transanal TME (TaTME) has been developed as an option to retain circumferential resection margins $(\mathrm{CRM})$ during TME. $^{46}$ The most common reconstruction method in lower anterior resection is the double-stapling technique (DST) anastomosis. Anastomotic leakage (AL) after low anterior resection for rectal cancer is a condition that can lead to serious complications, and it is reported to occur at a frequency of approximately 10 to $15 \%$ according to the National Clinical Database (NCD) in Japan and the Nationwide Inpatient Sample (NIS) database in the United States. ${ }^{47,}{ }^{48}$ Various 
factors such as anastomotic device, anastomotic blood flow, tension at the anastomotic site, and comorbidity are involved in the occurrence of AL. ${ }^{49-51}$ Furthermore, AL is related to a patient's quality of life and prognosis, especially morbidity, mortality, functional defects, and oncologic outcomes. ${ }^{52-54}$

\section{Laparoscopic surgery for advanced rectal cancer}

In rectal cancer, the role of conventional multiport laparoscopy is still debated. Most randomized and observational studies comparing laparoscopic and open resection for rectal cancer found equivalent oncologic margins, lymph node harvest, and long-term oncologic outcomes, except for the ACOSOG Z6051 and ALaCaRT randomized trials. ${ }^{55}$ The results of randomized trials could not prove that the short-term oncologic outcomes of laparoscopic surgery were equivalent to those after open surgery, even when performed by surgeons with laparoscopic expertise. ${ }^{55}$

\section{Robotic surgery for CRC}


The first robotic-assisted colectomy was performed in 2002 in the USA (Fig.5). ${ }^{56}$ Advantages of robotic surgery include tremor filtration, ability to scale motion, stereoscopic vision, and the use of wristed instruments. ${ }^{57}$ These improve the surgeon's dexterity and allow precise lymph node dissection and intracorporeal anastomoses. Cohort studies have shown that compared with laparoscopic approaches, robotic approaches for partial and total colectomies appear to offer improved long-term survival in patients with stage I-III colon cancer. ${ }^{58}$ However, prolonged operating times, increased costs, and steeper learning curves remain the major drawbacks of robotic colectomy for colon cancer. ${ }^{59}$ The role of robots in colon cancer surgery remains unclear. Conversely, in case of rectal cancer, robot-assisted laparoscopic TME and transanal minimally invasive TME might improve mid-rectal and distal rectal cancer outcomes. ${ }^{60,61}$ A metaanalysis showed that compared with laparoscopic surgery, robot-assisted surgery for rectal cancer may have a better therapeutic effect and similar oncological outcomes. ${ }^{62}$ Conversely, urinary and sexual dysfunction are potential complications of rectal cancer surgery; a prospective study noted that robotic surgery offered distinct advantages in 
terms of protecting the pelvic autonomic nerves and relieving postoperative sexual dysfunction. $^{63}$

\section{Navigation surgery}

Navigation technology has significantly contributed to enhancing, safe, and accurate surgery (Fig.5). In colorectal surgery, injecting fluorescence and indocyanine green (ICG) in the artery can be used for real-time visualization of blood flow of colon and rectum to be reconstructed. In addition, the incidence of AL due to blood flow insufficiency may be reduced by assessing the perfusion of a colorectal anastomosis. ${ }^{64}$ Furthermore, artificial intelligence (AI)-based real-time microcirculation analysis had a more accurate consistent performance than the conventional parameter-based method. ${ }^{65}$ Interestingly, ICG is also used for lymph node mapping. Sentinel lymph node mapping was developed to identify the first to fourth LNs in the lymphatic pathway, which are most likely to harbor micrometastases ${ }^{66}$ However, the fluorescent signal only indicates lymph node presence; therefore, tumor-specific targeting by CEA-targeted fluorescent imaging using SGM-101 is expected to be a tool for detecting the presence of lymph node metastasis. ${ }^{67}$ A complete 
understanding of the anatomy of blood vessels is vital for lymph node dissection and vascular resection for the treatment of colon cancer. Three-dimensional computed tomographic angiography with computed tomographic colonography may contribute to laparoscopic colorectal surgery by providing surgeons with reliable preoperative vascular anatomy. Preoperative information on vessel anatomy can help manipulate the blood vessels, prevent intraoperative vascular injury, and determine the appropriate extent of lymph node. ${ }^{68}$

\section{CHEMOTHERAPY AND RADIATION}

Chemotherapy for CRC is broadly classified as a treatment for neoadjuvant chemotherapy, adjuvant chemotherapy, and unresectable or recurrent cases. Commonly used anticancer drugs that have been approved for the treatment of CRC and are covered by the Japanese National Health Insurance include the followings ${ }^{8}$ :

Cytotoxic drugs: fluorouracil (5-FU), 5-FU + levofolinate calcium (l-LV), tegafur uracil (UFT), tegafur gimeracil oteracil potassium (S-1), UFT + calcium folinate, capecitabine, 
irinotecan hydrochloride hydrate, oxaliplatin (OX), trifluridine/tipiracil hydrochloride

(FTD/TPI).

Molecular targeted drugs: bevacizumab (BEV), ramucirumab (RAM), aflibercept beta

(AFL), cetuximab (CET), panitumumab (PANI), regorafenib hydrate (REG), encorafenib, binimetinib.

Immune checkpoint inhibitor: pembrolizumab (Pembro).

\section{Neoadjuvant therapy}

There are several benefits of neoadjuvant systemic therapy: (I) improvement of surgical outcomes by downstaging of tumor; (II) early control of systemic metastatic spread and testing of tumor biology; and (III) in vivo testing of chemotherapy sensitivity and potential incorporation of novel agents in clinical trials. ${ }^{69}$ Meta-analysis showed the potential survival benefit of neoadjuvant chemotherapy compared to adjuvant chemotherapy for locally advanced colon cancer, without an increase in surgical morbidity. ${ }^{69}$ Furthermore, preoperative chemotherapy in combination with chemoradiation, the so-called total neoadjuvant chemotherapy (TNT), has been reported 
to have the same benefits. ${ }^{70}$ A meta-analysis of the treatment outcomes of TNT was found to increase the odds of complete pathological response by $39 \% .^{71}$

\section{Adjuvant chemotherapy}

Postoperative adjuvant chemotherapy is systemic chemotherapy performed after surgery to prevent recurrence and improve the prognosis of patients who undergo R0 resection. As an adjuvant therapy, fluoropyrimidine-based chemotherapy improves survival in resected stage III, and in a subset of high-risk stage II colon cancers (e.g., T4 lesions, less than 12 examined lymph nodes, poorly differentiated histology). ${ }^{72,73}$ Several landmark studies, including the MOSAIC study, established the addition of oxaliplatin to fluoropyrimidine (fluorouracil or capecitabine) as a new standard. ${ }^{74-77}$ For years, 6 months of adjuvant chemotherapy is the standard of care. However, the IDEA collaboration, drawing on six randomized, phase 3 clinical trials, showed that limiting adjuvant chemotherapy to 3 months might reduce toxicity (e.g., less cumulative neuropathy) without impairing treatment efficacy for at least low-risk stage III colon cancers (not T4 or N2). ${ }^{78}$ 


\section{Chemotherapy for unresectable or recurrent CRC}

Systemic therapy for metastatic CRC is tailored with patient-specific and disease-specific predictive markers such as the RAS (KRAS/NRAS) and BRAF ${ }^{V 600 E}$ mutations and a DNAmismatch repair status. ${ }^{79}$ Paralleled with advances in surgical and allied specialties, the increasing number of effective drugs for CRC has substantially improved overall survival. The options for molecular stratified treatment available to patients with CRC have increased, and biomarkers have been used to determine the treatment strategy. ${ }^{80}$ Thus, the treatment options available to patients have expanded.

\section{Radiotherapy}

Radiotherapy is used to treat patients with locally advanced rectal cancer either as adjuvant therapy after surgery to prevent recurrence or before surgery to reduce tumor volume and preserve the anal sphincter, as well as palliative care to relieve the symptoms and prolong the survival time of patients with unresectable colorectal cancer who have symptomatic lesions. ${ }^{8}$ 


\section{CONCLUSIONS}

Currently, patients with advanced CRC are still mainly treated by surgery combined with neoadjuvant chemotherapy, adjuvant chemotherapy, and radiotherapy to improve surgical treatment. Sue to the features such as sharp dissection along the embryological planes within the mesofascial interface with full regional lymph node dissection, CME for colon cancer and TME for rectal cancer are recommended. With the advent of individualized and personalized medicine, continued improvements in the treatment of advanced CRC require supporting data from randomized clinical trials, which are also needed to provide evidence to support the extent of bowel resection and appropriate central lymphadenectomy in colon cancer surgery. Further studies are required to determine the optimal approaches for surgical treatment of patients with advanced CRC.

\section{REFERENCES}

1. Bray F, Ferlay J, Soerjomataram I, Siegel RL, Torre LA, Jemal A. Global cancer statistics 2018: GLOBOCAN estimates of incidence and mortality worldwide for 
36 cancers in 185 countries. CA Cancer J Clin. 2018 Nov;68(6):394-424.

2. Vogelstein B, Fearon ER, Hamilton SR, et al. Genetic alterations during colorectal-tumor development. N Engl J Med. 1988 Sep 1;319(9):525-32.

3. Crockett SD, Nagtegaal ID. Terminology, Molecular Features, Epidemiology, and Management of Serrated Colorectal Neoplasia. Gastroenterology. 2019 Oct;157(4):949-66 e4.

4. Thanikachalam K, Khan G. Colorectal Cancer and Nutrition. Nutrients. 2019 Jan $14 ; 11(1)$.

5. Botteri E, Iodice S, Bagnardi V, Raimondi S, Lowenfels AB, Maisonneuve P. Smoking and colorectal cancer: a meta-analysis. JAMA. 2008 Dec $17 ; 300(23): 2765-78$.

6. Pilleron S, Soto-Perez-de-Celis E, Vignat J, et al. Estimated global cancer incidence in the oldest adults in 2018 and projections to 2050. Int J Cancer. 2021 Feb 1;148(3):601-8.

7. Sehgal M, Ladabaum U, Mithal A, Singh H, Desai M, Singh G. Colorectal Cancer Incidence after Colonoscopy at Ages 45-49 or 50-54 Years. Gastroenterology. 
2021 Feb 9.

8. Hashiguchi Y, Muro K, Saito Y, et al. Japanese Society for Cancer of the Colon and Rectum (JSCCR) guidelines 2019 for the treatment of colorectal cancer. Int J Clin Oncol. 2020 Jan;25(1):1-42.

9. Goto O, Koizumi E, Higuchi K, et al. Cutting-Edge Technologies for Gastrointestinal Therapeutic Endoscopy. J Nippon Med Sch. 2021;88(1):17-24.

10. Toyonaga T, Ohara Y, Baba S, et al. Peranal endoscopic myectomy (PAEM) for rectal lesions with severe fibrosis and exhibiting the muscle-retracting sign. Endoscopy. 2018 Aug;50(8):813-7.

11. Schmidt A, Beyna T, Schumacher B, et al. Colonoscopic full-thickness resection using an over-the-scope device: a prospective multicentre study in various indications. Gut. 2018 Jul;67(7):1280-9.

12. Law R, Das A, Gregory D, et al. Endoscopic resection is cost-effective compared with laparoscopic resection in the management of complex colon polyps: an economic analysis. Gastrointest Endosc. 2016 Jun;83(6):1248-57.

13. Landry M, Cavalea AC, Bhat SG, Heidel RE, Casillas MA, Jr., Russ AJ. 
Combined Endoscopic and Laparoscopic Surgery versus Laparoscopic

Colectomy: Improved Patient Outcomes for Endoscopically Unresectable

Neoplasms. Am Surg. 2020 Mar 1;86(3):e164-e6.

14. Kudo SE, Ichimasa K, Villard B, et al. Artificial Intelligence System to Determine

Risk of T1 Colorectal Cancer Metastasis to Lymph Node. Gastroenterology. 2021

Mar;160(4):1075-84 e2.

15. Poston GJ, Figueras J, Giuliante F, et al. Urgent need for a new staging system in advanced colorectal cancer. J Clin Oncol. 2008 Oct 10;26(29):4828-33.

16. Kobayashi H, West NP. CME versus D3 Dissection for Colon Cancer. Clin Colon Rectal Surg. 2020 Nov;33(6):344-8.

17. Hohenberger W, Weber K, Matzel K, Papadopoulos T, Merkel S. Standardized surgery for colonic cancer: complete mesocolic excision and central ligation-technical notes and outcome. Colorectal Dis. 2009 May;11(4):354-64; discussion 64-5.

18. Garcia-Granero A, Pellino G, Frasson M, et al. The fusion fascia of Fredet: an important embryological landmark for complete mesocolic excision and D3- 
lymphadenectomy in right colon cancer. Surg Endosc. 2019 Nov;33(11):3842-50.

19. Wang C, Gao Z, Shen K, et al. Safety, quality and effect of complete mesocolic excision vs non-complete mesocolic excision in patients with colon cancer: a systemic review and meta-analysis. Colorectal Dis. 2017 Nov;19(11):962-72.

20. Emmanuel A, Haji A. Complete mesocolic excision and extended (D3) lymphadenectomy for colonic cancer: is it worth that extra effort? A review of the literature. Int J Colorectal Dis. 2016 Apr;31(4):797-804.

21. Shiozawa M, Ueno H, Shiomi A, et al. Study protocol for an International Prospective Observational Cohort Study for Optimal Bowel Resection Extent and Central Radicality for Colon Cancer (T-REX study). Jpn J Clin Oncol. 2021 Jan $1 ; 51(1): 145-55$.

22. Phillips EH, Franklin M, Carroll BJ, Fallas MJ, Ramos R, Rosenthal D. Laparoscopic colectomy. Ann Surg. 1992 Dec;216(6):703-7.

23. Colon Cancer Laparoscopic or Open Resection Study G, Buunen M, Veldkamp R, et al. Survival after laparoscopic surgery versus open surgery for colon cancer: long-term outcome of a randomised clinical trial. Lancet Oncol. 2009 
Jan;10(1):44-52.

24. Jayne DG, Guillou PJ, Thorpe H, et al. Randomized trial of laparoscopic-assisted resection of colorectal carcinoma: 3-year results of the UK MRC CLASICC Trial Group. J Clin Oncol. 2007 Jul 20;25(21):3061-8.

25. Green BL, Marshall HC, Collinson F, et al. Long-term follow-up of the Medical Research Council CLASICC trial of conventional versus laparoscopically assisted resection in colorectal cancer. Br J Surg. 2013 Jan;100(1):75-82.

26. Fleshman J, Sargent DJ, Green E, et al. Laparoscopic colectomy for cancer is not inferior to open surgery based on 5-year data from the COST Study Group trial. Ann Surg. 2007 Oct;246(4):655-62; discussion 62-4.

27. Lacy AM, Delgado S, Castells A, et al. The long-term results of a randomized clinical trial of laparoscopy-assisted versus open surgery for colon cancer. Ann Surg. 2008 Jul;248(1):1-7.

28. Shida D, Ochiai H, Tsukamoto S, Kanemitsu Y. Long-term outcomes of laparoscopic versus open D3 dissection for stage II/III colon cancer: Results of propensity score analyses. Eur J Surg Oncol. 2018 Jul;44(7):1025-30. 
29. Kitano S, Inomata M, Mizusawa J, et al. Survival outcomes following laparoscopic versus open D3 dissection for stage II or III colon cancer (JCOG0404): a phase 3, randomised controlled trial. Lancet Gastroenterol Hepatol. 2017 Apr;2(4):261-8.

30. Mar J, Anton-Ladislao A, Ibarrondo O, et al. Cost-effectiveness analysis of laparoscopic versus open surgery in colon cancer. Surg Endosc. 2018 Dec;32(12):4912-22.

31. Maruyama H, Kusachi S, Makino H, Kanno H, Yoshida H, Niitsuma T. Postoperative Infection after Colorectal Surgery: Subanalysis of Data from the 2015 Japan Postoperative Infectious Complications Survey. J Nippon Med Sch. 2020 Sep 9;87(4):204-10.

32. Quintana JM, Anton-Ladisla A, Gonzalez N, et al. Outcomes of open versus laparoscopic surgery in patients with colon cancer. Eur J Surg Oncol. 2018 Sep;44(9):1344-53.

33. Gavriilidis P, Katsanos K. Laparoscopic Versus Open Transverse Colectomy: A Systematic Review and Meta-Analysis. World J Surg. 2018 Sep;42(9):3008-14. 
34. Toritani K, Watanabe J, Nakagawa K, et al. Randomized controlled trial to evaluate laparoscopic versus open surgery in transverse and descending colon cancer patients. Int J Colorectal Dis. 2019 Jul;34(7):1211-20.

35. Matsuda T, Sumi Y, Yamashita K, et al. Optimal Surgery for Mid-Transverse Colon Cancer: Laparoscopic Extended Right Hemicolectomy Versus Laparoscopic Transverse Colectomy. World J Surg. 2018 Oct;42(10):3398-404.

36. Heald RJ, Husband EM, Ryall RD. The mesorectum in rectal cancer surgery--the clue to pelvic recurrence? Br J Surg. 1982 Oct;69(10):613-6.

37. Heald RJ, Moran BJ, Ryall RD, Sexton R, MacFarlane JK. Rectal cancer: the Basingstoke experience of total mesorectal excision, 1978-1997. Arch Surg. 1998 Aug;133(8):894-9.

38. Adam IJ, Mohamdee MO, Martin IG, et al. Role of circumferential margin involvement in the local recurrence of rectal cancer. Lancet. 1994 Sep 10;344(8924):707-11.

39. Nagtegaal ID, Marijnen CA, Kranenbarg EK, et al. Circumferential margin involvement is still an important predictor of local recurrence in rectal carcinoma: 
not one millimeter but two millimeters is the limit. Am J Surg Pathol. 2002 Mar;26(3):350-7.

40. Bernstein TE, Endreseth BH, Romundstad P, Wibe A, Norwegian Colorectal Cancer R. Improved local control of rectal cancer reduces distant metastases. Colorectal Dis. 2012 Oct;14(10):e668-78.

41. Colvin H, Mizushima T, Eguchi H, Takiguchi S, Doki Y, Mori M. Gastroenterological surgery in Japan: The past, the present and the future. Ann Gastroenterol Surg. 2017 Apr;1(1):5-10.

42. Cunningham D, Atkin W, Lenz HJ, et al. Colorectal cancer. Lancet. 2010 Mar 20;375(9719):1030-47.

43. Fujita S, Mizusawa J, Kanemitsu Y, et al. Mesorectal Excision With or Without Lateral Lymph Node Dissection for Clinical Stage II/III Lower Rectal Cancer (JCOG0212): A Multicenter, Randomized Controlled, Noninferiority Trial. Ann Surg. 2017 Aug;266(2):201-7.

44. Tsukamoto S, Fujita S, Ota M, et al. Long-term follow-up of the randomized trial of mesorectal excision with or without lateral lymph node dissection in rectal 
cancer (JCOG0212). Br J Surg. 2020 Apr;107(5):586-94.

45. Wang X, Qiu A, Liu X, Shi Y. Total mesorectal excision plus lateral lymph node dissection vs TME on rectal cancer patients: a meta-analysis. Int J Colorectal Dis. 2020 Jun;35(6):997-1006.

46. Penna M, Hompes R, Arnold S, et al. Transanal Total Mesorectal Excision: International Registry Results of the First 720 Cases. Ann Surg. 2017 Jul;266(1):111-7.

47. Watanabe T, Miyata H, Konno H, et al. Prediction model for complications after low anterior resection based on data from 33,411 Japanese patients included in the National Clinical Database. Surgery. 2017 Jun;161(6):1597-608.

48. Kang CY, Halabi WJ, Chaudhry OO, et al. Risk factors for anastomotic leakage after anterior resection for rectal cancer. JAMA Surg. 2013 Jan;148(1):65-71.

49. Braunschmid T, Hartig N, Baumann L, Dauser B, Herbst F. Influence of multiple stapler firings used for rectal division on colorectal anastomotic leak rate. Surg Endosc. 2017 Dec;31(12):5318-26.

50. Kawada K, Sakai Y. Preoperative, intraoperative and postoperative risk factors for 
anastomotic leakage after laparoscopic low anterior resection with double stapling technique anastomosis. World J Gastroenterol. 2016 Jul 7;22(25):5718-27.

51. Shinji S, Ueda Y, Yamada T, et al. Male sex and history of ischemic heart disease are major risk factors for anastomotic leakage after laparoscopic anterior resection in patients with rectal cancer. BMC Gastroenterol. 2018 Jul 17;18(1):117.

52. Walker KG, Bell SW, Rickard MJ, et al. Anastomotic leakage is predictive of diminished survival after potentially curative resection for colorectal cancer. Ann Surg. 2004 Aug;240(2):255-9.

53. Bell SW, Walker KG, Rickard MJ, et al. Anastomotic leakage after curative anterior resection results in a higher prevalence of local recurrence. Br J Surg. 2003 Oct;90(10):1261-6.

54. Hallbook O, Sjodahl R. Anastomotic leakage and functional outcome after anterior resection of the rectum. Br J Surg. 1996 Jan;83(1):60-2.

55. Cleary RK, Morris AM, Chang GJ, Halverson AL. Controversies in Surgical Oncology: Does the Minimally Invasive Approach for Rectal Cancer Provide Equivalent Oncologic Outcomes Compared with the Open Approach? Ann Surg 
Oncol. 2018 Nov;25(12):3587-95.

56. Weber PA, Merola S, Wasielewski A, Ballantyne GH. Telerobotic-assisted laparoscopic right and sigmoid colectomies for benign disease. Dis Colon Rectum. 2002 Dec;45(12):1689-94; discussion 95-6.

57. Giulianotti PC, Coratti A, Angelini M, et al. Robotics in general surgery: personal experience in a large community hospital. Arch Surg. 2003 Jul;138(7):777-84.

58. Mirkin KA, Kulaylat AS, Hollenbeak CS, Messaris E. Robotic versus laparoscopic colectomy for stage I-III colon cancer: oncologic and long-term survival outcomes. Surg Endosc. 2018 Jun;32(6):2894-901.

59. Isik O, Gorgun E. How Has the Robot Contributed to Colon Cancer Surgery? Clin Colon Rectal Surg. 2015 Dec;28(4):220-7.

60. Jayne D, Pigazzi A, Marshall H, et al. Effect of Robotic-Assisted vs Conventional Laparoscopic Surgery on Risk of Conversion to Open Laparotomy Among Patients Undergoing Resection for Rectal Cancer: The ROLARR Randomized Clinical Trial. JAMA. 2017 Oct 24;318(16):1569-80.

61. Ma B, Gao P, Song Y, et al. Transanal total mesorectal excision (taTME) for rectal 
cancer: a systematic review and meta-analysis of oncological and perioperative outcomes compared with laparoscopic total mesorectal excision. BMC Cancer. 2016 Jul 4;16:380.

62. Wang X, Cao G, Mao W, Lao W, He C. Robot-assisted versus laparoscopic surgery for rectal cancer: A systematic review and meta-analysis. J Cancer Res Ther. 2020 Sep;16(5):979-89.

63. Wang G, Wang Z, Jiang Z, Liu J, Zhao J, Li J. Male urinary and sexual function after robotic pelvic autonomic nerve-preserving surgery for rectal cancer. Int $\mathrm{J}$ Med Robot. 2017 Mar;13(1).

64. Arezzo A, Bonino MA, Ris F, et al. Intraoperative use of fluorescence with indocyanine green reduces anastomotic leak rates in rectal cancer surgery: an individual participant data analysis. Surg Endosc. 2020 Oct;34(10):4281-90.

65. Park SH, Park HM, Baek KR, Ahn HM, Lee IY, Son GM. Artificial intelligence based real-time microcirculation analysis system for laparoscopic colorectal surgery. World J Gastroenterol. 2020 Nov 28;26(44):6945-62.

66. Borgstein PJ, Pijpers R, Comans EF, van Diest PJ, Boom RP, Meijer S. Sentinel 
lymph node biopsy in breast cancer: guidelines and pitfalls of lymphoscintigraphy and gamma probe detection. J Am Coll Surg. 1998 Mar;186(3):275-83.

67. Vuijk FA, Hilling DE, Mieog JSD, Vahrmeijer AL. Fluorescent-guided surgery for sentinel lymph node detection in gastric cancer and carcinoembryonic antigen targeted fluorescent-guided surgery in colorectal and pancreatic cancer. J Surg Oncol. 2018 Aug;118(2):315-23.

68. Hiroishi A, Yamada T, Morimoto T, Horikoshi K, Nakajima Y. Three-dimensional computed tomographic angiography with computed tomographic colonography for laparoscopic colorectal surgery. Jpn J Radiol. 2018 Dec;36(12):698-705.

69. Cheong CK, Nistala KRY, $\mathrm{Ng} \mathrm{CH}$, et al. Neoadjuvant therapy in locally advanced colon cancer: a meta-analysis and systematic review. J Gastrointest Oncol. 2020 Oct;11(5):847-57.

70. Cercek A, Roxburgh CSD, Strombom P, et al. Adoption of Total Neoadjuvant Therapy for Locally Advanced Rectal Cancer. JAMA Oncol. 2018 Jun 14;4(6):e180071.

71. Petrelli F, Trevisan F, Cabiddu M, et al. Total Neoadjuvant Therapy in Rectal 
Cancer: A Systematic Review and Meta-analysis of Treatment Outcomes. Ann Surg. 2020 Mar;271(3):440-8.

72. Ishiguro M, Ueno $\mathrm{H}$, Kanemitsu $\mathrm{Y}$, et al. Current clinical practice of adjuvant chemotherapy for patients with 'high-risk' Stage II colorectal cancer in Japan: a questionnaire survey in the JCOG Study Group. Jpn J Clin Oncol. 2018 Dec 1;48(12):1109-12.

73. Enofe N, Morris AD, Liu Y, et al. Receipt of Adjuvant Chemotherapy in Stage II Colon Cancer and Overall Survival: A National Cancer Database Study. J Surg Res. 2020 Aug;252:69-79.

74. Kuebler JP, Wieand HS, O'Connell MJ, et al. Oxaliplatin combined with weekly bolus fluorouracil and leucovorin as surgical adjuvant chemotherapy for stage II and III colon cancer: results from NSABP C-07. J Clin Oncol. 2007 Jun $1 ; 25(16): 2198-204$.

75. Andre T, Boni C, Navarro M, et al. Improved overall survival with oxaliplatin, fluorouracil, and leucovorin as adjuvant treatment in stage II or III colon cancer in the MOSAIC trial. J Clin Oncol. 2009 Jul 1;27(19):3109-16. 
76. Haller DG, Tabernero J, Maroun J, et al. Capecitabine plus oxaliplatin compared with fluorouracil and folinic acid as adjuvant therapy for stage III colon cancer. J Clin Oncol. 2011 Apr 10;29(11):1465-71.

77. Yothers G, O'Connell MJ, Allegra CJ, et al. Oxaliplatin as adjuvant therapy for colon cancer: updated results of NSABP C-07 trial, including survival and subset analyses. J Clin Oncol. 2011 Oct 1;29(28):3768-74.

78. Grothey A, Sobrero AF, Shields AF, et al. Duration of Adjuvant Chemotherapy for Stage III Colon Cancer. N Engl J Med. 2018 Mar 29;378(13):1177-88.

79. Guo TA, Wu YC, Tan C, et al. Clinicopathologic features and prognostic value of KRAS, NRAS and BRAF mutations and DNA mismatch repair status: A singlecenter retrospective study of 1,834 Chinese patients with Stage I-IV colorectal cancer. Int J Cancer. 2019 Sep 15;145(6):1625-34.

80. Sveen A, Kopetz S, Lothe RA. Biomarker-guided therapy for colorectal cancer: strength in complexity. Nat Rev Clin Oncol. 2020 Jan;17(1):11-32. 


\section{FIGURE LEGENDS}

Figure 1. Endoscopic mucosal resection is used to resect precancerous, early-stage cancer from the digestive tract. It is performed by injecting saline solution into the submucosal space to elevate the lesion (A). Subsequently, the lesion is captured and removed using a snare while minimizing mechanical or electrocautery damage to the deeper layers of the gastrointestinal wall $(\mathrm{B}, \mathrm{C})$. The lesion may be removed in a single attempt or in a piecemeal fashion (D).

Figure 2. Endoscopic submucosal resection is used to remove large tumors from the digestive tract. Several marking dots are made outside the lesion (A), and a solution is injected submucosally (B). An incision is made around the perimeter of the diseased part or lesion using a cutting knife (C), and a direct dissection of the submucosal layer is performed (D).

Figure 3. Endoscopic full-thickness resection is a novel treatment for colorectal lesions that are not amenable to conventional endoscopic resection. A pair of grasping forceps or 
an anchor device (Ovesco Endoscopy) is advanced through the working channel (A). The lesion is then gently pulled into the cap (B). A clip is then deployed, and the tissue above the clip is immediately resected with a snare (C).

Figure 4. An illustration shows the embryological plane defined by the fusion fascia of Toldt and the fusion fascia of Fredet, which exist between the right mesocolon and the retroperitoneum.

Figure 5. The evolution of surgery for CRC. In the early 1990s, surgeons switched from open surgery to laparoscopic surgery. In the 2000s, robotic surgery was added to laparoscopic surgery. Recently, the development of artificial intelligence-based technology and navigation surgery has been advancing rapidly. 
Acknowledgements: We would like to thank Editage (https://www.editage.com).

Conflict of Interest: The authors declare no competing interests. 
Fig. 1
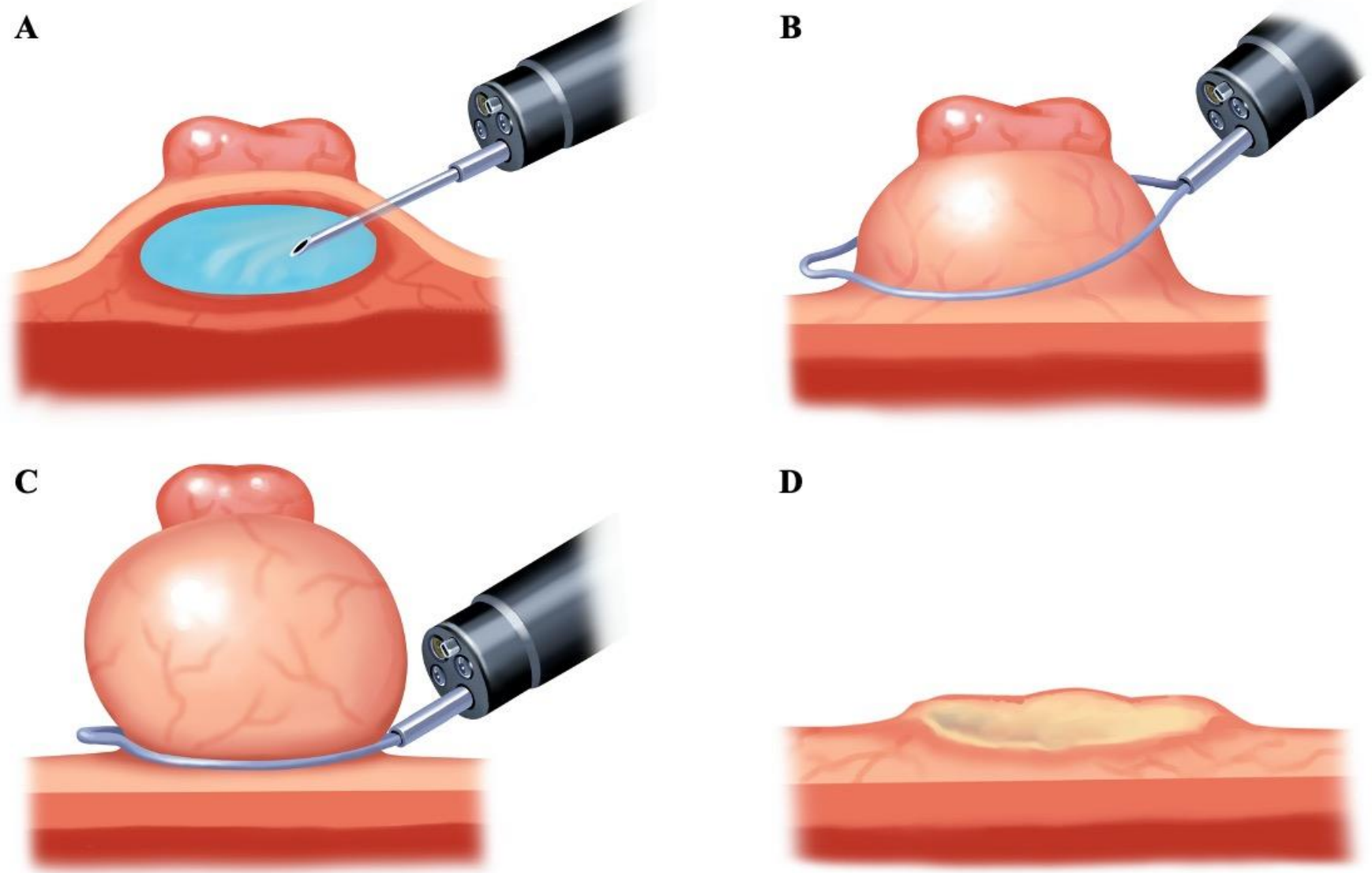

D

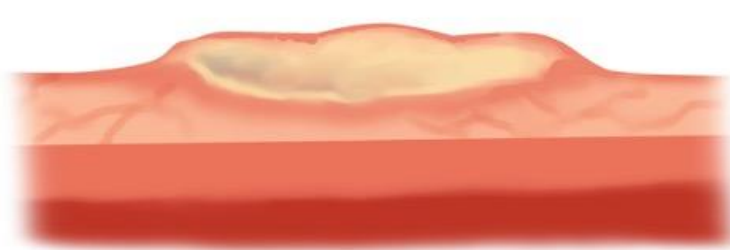


Fig. 2

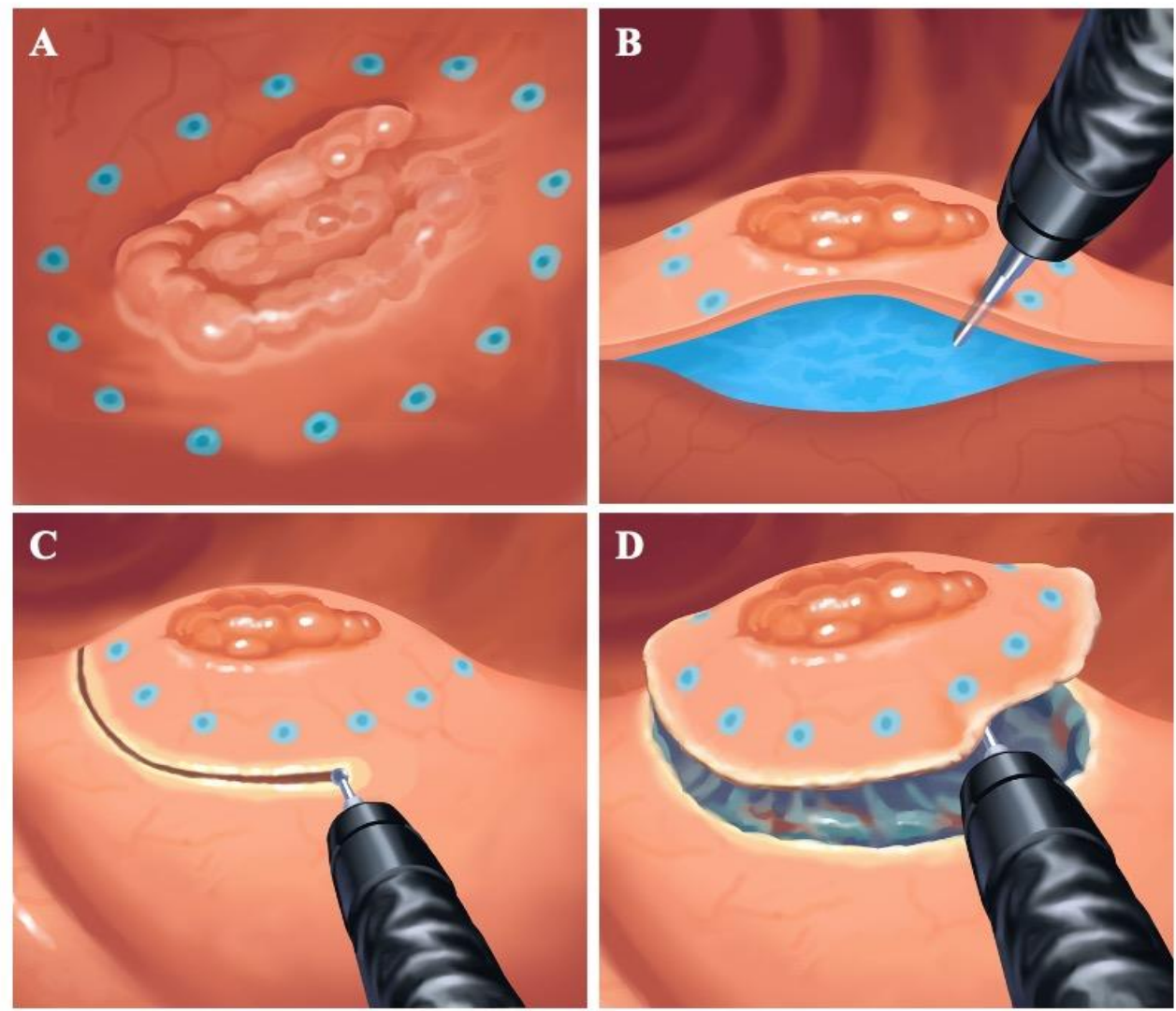


Fig. 3

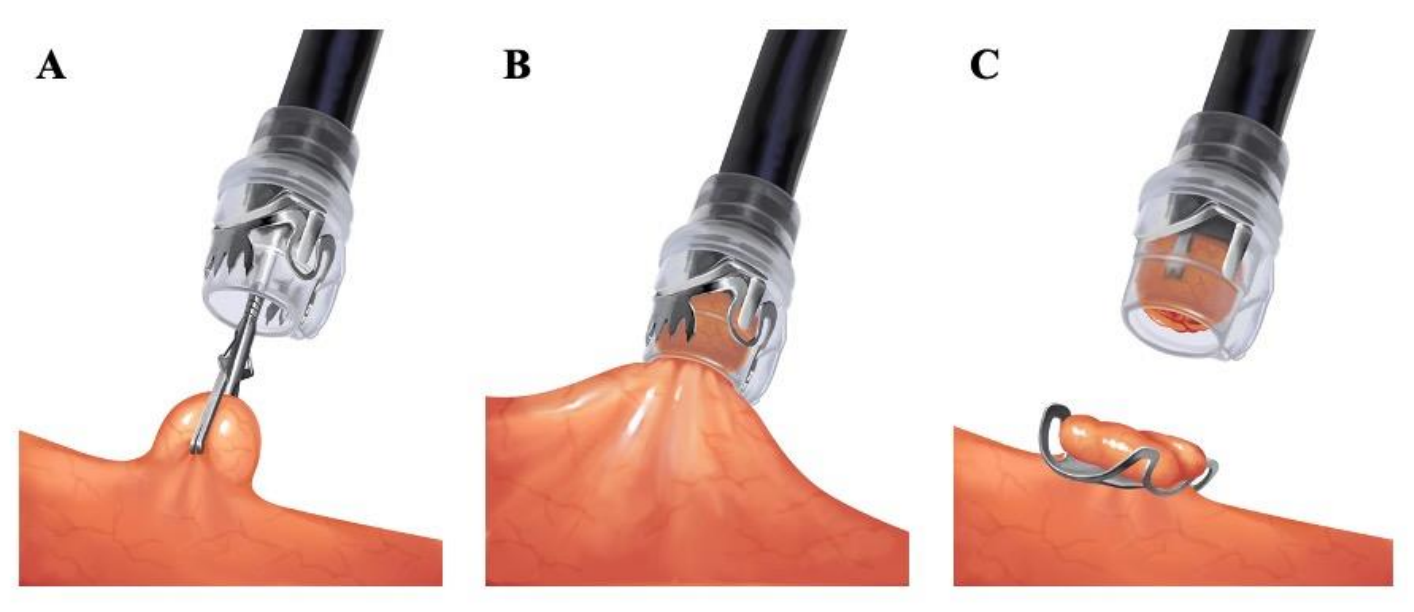


Fig. 4

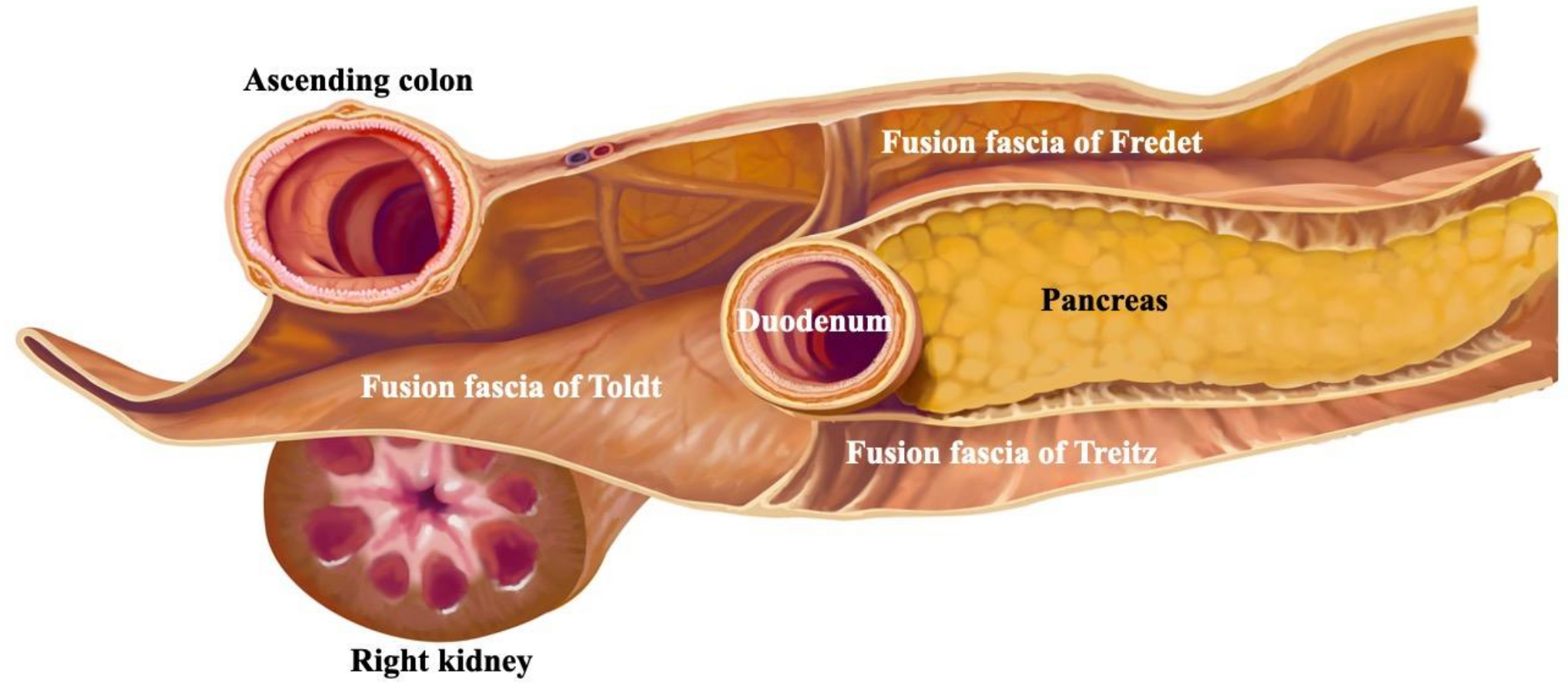


Fig. 5
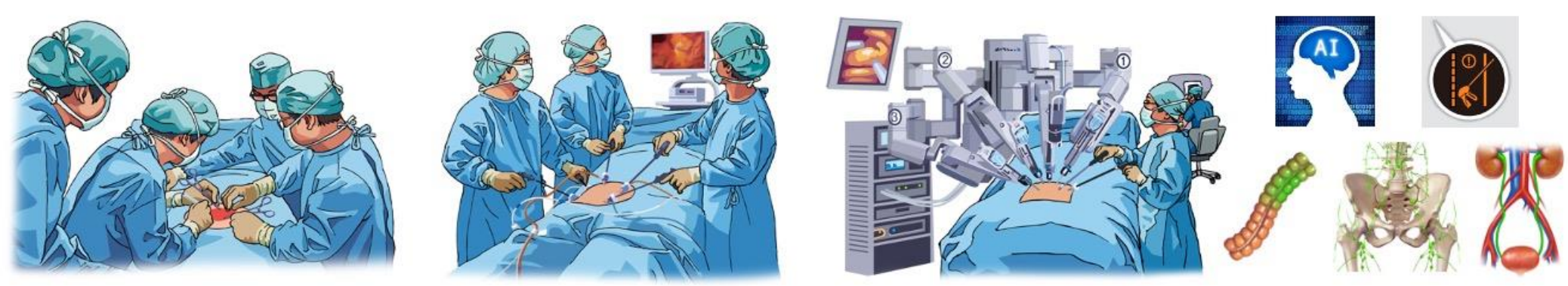

-1990 's

1990’s

2000's

2020's 